\title{
Active structural acoustic control of broadband disturbances
}

\author{
William T. Baumann, Fu-Sheng Ho, and Harry H. Robertshaw \\ Interdisciplinary Center for Applied Mathematics, Center for Intelligent Material Systems and Structures, \\ Virginia Polytechnic Institute and State University, Blackburg, Virginia 24061
}

(Received 17 July 1991; revised 7 January 1992; accepted 2 June 1992)

\begin{abstract}
A control design technique is developed to actively suppress the acoustic power radiated from a structure, with negligible fluid loading, that is persistently excited by narrow-band or broadband disturbances. The problem is constrained by the assumption that the far-field pressure cannot be measured directly. A method for estimating the total radiated power from measurements on the structure is developed. Using this estimate as a cost function and assuming knowledge of the spectrum of the disturbance, a controller is designed using the linear-quadratic-Gaussian (LQG) theory to minimize the cost. Computer simulations of a clamped-clamped beam show that there is a significant difference in the total radiated power between a system with a vibration-suppression controller and a system with an acoustic controller that accounts for the coupling of these vibrations to the surrounding fluid. In some cases, the acoustic controller increases the system vibration in order to minimize the radiated power.
\end{abstract}

PACS numbers: $43.40 . V n$

\section{INTRODUCTION}

Much of the work in the area of active structural acoustic control (ASAC) has involved the use of feedforward control for the suppression of harmonic disturbances. ${ }^{1,2}$ In practice, the controller is often implemented by an adaptive algorithm and requires almost no modeling of the structure. The successful extension of this work to broadband disturbances, however, is model based and requires accurate modeling of the structure over the disturbance bandwidth. ${ }^{3}$

In this paper we take a different approach and consider a nonadaptive, model-based, feedback approach to ASAC that can reduce the radiation due to persistent broadband disturbances as well as that due to transients arising from short duration excitations. Model-based ASAC has also been considered by Meirovitch and Thangjitham, ${ }^{4,5}$ but their approach is based on vibration control of harmonic disturbances, where the disturbance can be measured, and the vibration cost function is tuned during the design process to reduce the observed far-field pressure.

The contribution of this paper is to formulate the acoustic suppression problem for persistent broadband disturbances as a standard linear-quadratic-Gaussian (LQG) problem that can be solved by modern control methods. Computer simulations of a baffled, clamped-clamped beam example show that there is a significant difference in the total radiated power between a system with a vibration-suppression controller and a system with an acoustic controller that accounts for the coupling of these vibrations to the surrounding fluid. In some cases, the acoustic controller increases the system vibration in order to minimize the radiated power.

\section{ESTIMATING TOTAL RADIATED POWER}

We assume that the structural motion can be accurately modeled by a first-order ordinary differential equation of the form

$$
\dot{w}=A w+B u+L d,
$$

where $w \in R^{2 N}$ is the state of the system, $u \in R^{m}$ is a vector of actuator inputs, and $d \in R^{p}$ is the disturbance input, which will be assumed to be a wide sense stationary (WSS) random process. Such a model implicitly assumes that we have decomposed the structure spatially using a set of spatial functions, $\Phi_{i}(x)$.The spatial functions may be modes, finite elements, or any other convenient functions. In our examples, standard modal models are used. We assume that the state space coordinates are chosen such that the velocity of the structure at location $x$ and time $t$ is given by

$$
v(x, t)=\sum_{i=1}^{N} \Phi_{i}(x) w_{2 i}(t) .
$$

As discussed in Ref. 6, which dealt with the transient case, we can define a transfer function between each velocity component, $w_{2 i}(t)$, associated with spatial component $\Phi_{i}(x)$, and the pressure in the far field at the point $(R, \theta, \phi)$ as

$$
\frac{\hat{p}(R, \theta, \phi, \omega)}{\hat{w}_{2 i}(\omega)}=\frac{\hat{h}_{i}(\theta, \phi, \omega) e^{j k R}}{R} .
$$

The caret denotes the Fourier transform of a variable and $k=w / c$, where $c$ is the speed of sound in the fluid medium. The fact that such a transfer function exists follows from Rayleigh's integral formula. For simple systems, e.g., a beam, ${ }^{7}$ it can be computed in closed form. For more complex systems it must be evaluated numerically. Defining

$$
H(\theta, \phi, \omega)=\left[\begin{array}{llll}
\hat{h}_{1} & \hat{h}_{2} & \cdots & \hat{h}_{N}
\end{array}\right] ; \quad V(\omega)=\left[\begin{array}{c}
\hat{w}_{2} \\
\hat{w}_{4} \\
\vdots \\
\hat{w}_{2 N}
\end{array}\right],
$$

the total far-field pressure can be written as

$$
\hat{p}(R, \theta, \phi, \omega)=H(\theta, \phi, \omega) \cdot V(\omega)\left(e^{j k R} / R\right) .
$$


The average power radiated in the direction $(\theta, \phi)$ can be written in terms of the real-valued pressure as

$$
\lim _{T \rightarrow \infty} \frac{1}{\rho c T} \int_{0}^{T} p(R, \theta, \phi, t)^{2} d t .
$$

The pressure is a WSS random process since it is the output of a linear system with a WSS input. Anticipating Sec. II, where we will be interested in obtaining an optimal control that is independent of the particular sample function of the disturbance process, we consider the expected value of the radiated power, that is,

$$
\begin{aligned}
\Pi_{\theta, \phi} & =\lim _{T \rightarrow \infty} \frac{1}{\rho c T} E\left\{\int_{0}^{T} p(R, \theta, \phi, t)^{2} d t\right\} \\
& =(1 / \rho c) E\left\{p(R, \theta, \phi, t)^{2}\right\}
\end{aligned}
$$

where the second equality follows from the fact that $p$ is WSS. In terms of the power spectral density of $p(R, \theta, \phi, t)$, denoted by $S_{p}(R, \theta, \phi, \omega)$, the expected value of the radiated power can be written as

$$
\Pi_{\theta, \phi}=\frac{1}{2 \pi \rho c} \int_{-\infty}^{+\infty} S_{p}(R, \theta, \phi, \omega) d \omega .
$$

Since the far-field pressure can be thought of as the result of passing the modal velocity components $V$ through a linear system $H$, the power spectral density of the far-field pressure is related to that of the structural velocity components $S_{V}$ by $^{8}$

$$
\begin{aligned}
S_{p}(R, \theta, \phi, \omega) & =\frac{H(\theta, \phi, \omega) S_{V}(\omega) H^{T}(\theta, \phi,-\omega)}{R^{2}} \\
& =\frac{\operatorname{tr}\left\{S_{V}(\omega) H^{T^{*}}(\theta, \phi, \omega) H(\theta, \phi, \omega)\right\}}{R^{2}} .
\end{aligned}
$$

The expected value of the total radiated power is given by

$$
\begin{aligned}
\Pi & =\int_{0}^{2 \pi} \int_{0}^{\pi} \Pi_{\theta, \phi} R^{2} \sin \theta d \theta d \phi \\
& =\frac{1}{2 \pi} \int_{-\infty}^{+\infty} \operatorname{tr}\left\{S_{V}(\omega) M(\omega)\right\} d \omega,
\end{aligned}
$$

where

$$
M(\omega)=\frac{1}{\rho c} \int_{0}^{2 \pi} \int_{0}^{\pi} H^{T^{*}}(\theta, \phi, \omega) H(\theta, \phi, \omega) \sin \theta d \theta d \phi .
$$

As discussed in Ref. 6, we can factor $M(\omega)$ as

$$
M(\omega)=G^{T^{*}}(\omega) G(\omega),
$$

which allows us to write

$$
\begin{aligned}
\Pi & =\frac{1}{2 \pi} \int_{-\infty}^{+\infty} \operatorname{tr}\left\{G(\omega) S_{V}(\omega) G^{T^{*}}(\omega)\right\} d \omega \\
& =\frac{1}{2 \pi} \int_{-\infty}^{+\infty} \operatorname{tr}\left\{S_{z}(\omega)\right\} d \omega \\
& =\lim _{T \rightarrow \infty} \frac{E\left\{\int_{0}^{T} z^{T}(t) z(t) d t\right\}}{T},
\end{aligned}
$$

where $z(s)=G(s) V(s)$ (in the Laplace transform domain) is the result of passing the velocity components through a "radiation filter" with transfer function $G(s)$. Thus, we have created an auxiliary state $z$ whose average expected power is equal to the average expected value of the power radiated to the far field. Had we been concerned with a deterministic power-signal input, the results would be the same as the above without the expected value operator.

\section{CONTROL LAW DESIGN}

The physical system is modeled by the equations

$$
\begin{aligned}
& \dot{w}=A w+B u+L d, \\
& y=C w+n,
\end{aligned}
$$

where $y$ denotes the output of sensors on the system and $n$ is a white, Gaussian, measurement noise process. The disturbance input $d$ will be modeled as the result of passing a Gaussian white noise process $v$ through a linear shaping filter, which results in the equations

$$
\begin{aligned}
& \dot{s}=A_{D} s+B_{D} v, \\
& d=C_{D} s .
\end{aligned}
$$

The radiation filter $G(s)$ can be modeled in state space with equations of the form

$$
\begin{aligned}
& \dot{r}=A_{F} r+B_{F} w, \\
& z=C_{F} r+D_{F} w .
\end{aligned}
$$

The filter is driven by the velocity components of the state $w$ and this fact is accounted for in the form of the matrices $B_{F}$ and $D_{F}$. The filter is not driven directly by the actual measurements, $y$, which may consist of position, velocity, or acceleration measurements.

By defining an augmented state as $x=\left[\begin{array}{lll}w & r & s\end{array}\right]^{T}$, the overall system can be written in the state space form

$$
\begin{aligned}
\dot{x} & =\left[\begin{array}{ccc}
A & 0 & L C_{D} \\
B_{F} & A_{F} & 0 \\
0 & 0 & A_{D}
\end{array}\right] x+\left[\begin{array}{l}
B \\
0 \\
0
\end{array}\right] u+\left[\begin{array}{c}
0 \\
0 \\
B_{D}
\end{array}\right] v \\
& \triangleq A_{a} x+B_{a} u+L_{a} v, \\
y & =\left[\begin{array}{lll}
C & 0 & 0
\end{array}\right] x+n \triangleq C_{a} x+n, \\
z & =\left[\begin{array}{lll}
D_{F} & C_{F} & 0
\end{array}\right] x \triangleq H_{a} x .
\end{aligned}
$$

Consider a cost function of the form

$$
J=\lim _{T \rightarrow \infty} \frac{E\left\{\int_{0}^{T} z^{T}(t) z(t)+\rho u^{T} u d t\right\}}{T} .
$$

This differs from the expected total radiated power by the addition of a control power term. The parameter $\rho$ allows the control designer to trade off acoustic suppression with control power. The problem of optimally designing an outputfeedback controller to minimize this cost function for the system (4) is in the form of a standard linear-quadraticGaussian (LQG) control problem. The solution involves a Kalman filter of the form

$$
\dot{\hat{x}}=A_{a} \hat{x}+B_{a} u+K_{f}\left(y-C_{a} \hat{x}\right)
$$

coupled with feedback of the estimated state

$$
u=-K_{l q} \hat{x} \text {. }
$$

If the objective is to suppress the vibrations on the structure due to the persistent disturbance, a cost function of the form 


$$
J=\lim _{T \rightarrow \infty} \frac{E\left\{\int_{0}^{T} w^{T}(t) Q w(t)+\rho u^{T} u d t\right\}}{T} .
$$

would be used. The controller would have the same form, but the state feedback gain $K_{l q}$ would differ.

The overall closed-loop system satisfies the equations

$$
\begin{aligned}
\frac{d}{d t}\left[\begin{array}{l}
w \\
\hat{x}
\end{array}\right]= & {\left[\begin{array}{cc}
A & -B K_{l q} \\
K_{f} C & A_{a}-B_{a} K_{l q}-K_{f} C_{a}
\end{array}\right]\left[\begin{array}{l}
w \\
\hat{x}
\end{array}\right] } \\
& +\left[\begin{array}{l}
L \\
0
\end{array}\right] d+\left[\begin{array}{c}
0 \\
K_{f}
\end{array}\right] n .
\end{aligned}
$$

\section{EXAMPLE}

Consider an Euler-Bernoulli model for a uniform bar in a clamped-clamped configuration,

$$
\begin{aligned}
& E I \frac{\partial^{4} y(x, t)}{\partial x^{4}}+m \frac{\partial^{2} y(x, t)}{\partial t^{2}}=f(x, t), \\
& y(0, t)=y(L, t)=0 ; \quad \frac{\partial y(0, t)}{\partial x}=\frac{\partial y(L, t)}{\partial x}=0,
\end{aligned}
$$

where $y$ is the displacement and $f$ is the force per unit length. The parameters of the beam are

$\begin{array}{ll}\text { Young's modulus, } E & 2.04 \times 10^{11} \mathrm{~Pa}, \\ \text { mass per unit length, } m & 0.491 \mathrm{~kg} / \mathrm{m}, \\ \text { length, } L & 1.0 \mathrm{~m}, \\ \text { width, } b & 0.125 \mathrm{~m}, \\ \text { thickness, } h & 0.5 \mathrm{~mm},\end{array}$

and the inertia $I$ is computed using $I=b h^{3} / 12$.

The mode shapes are given by ${ }^{7}$

$$
\Phi_{i}(x)=\cos k_{i} x-\cosh k_{i} x+R_{i}\left(\sin k_{i} x-\sinh k_{i} x\right)
$$

where

$$
R_{i}=\left(\cos k_{i} L-\cosh k_{i} L\right) /\left(\sinh k_{i} L-\sin k_{i} L\right)
$$

and $k_{i} L$ is the $i$ th root of

$$
\cosh k_{i} L \cos k_{i} L=1 \text {. }
$$

This information allows us to construct a state space model for the open-loop system once the locations of the disturbance and control forces have been specified. For simplicity, we assume that the three modal velocities are directly measured and the covariance of the measurement noise is $10^{-10}$.

The beam is mounted in a baffle and we are concerned with suppressing radiation into a half-space. Thus the integral in (2) will be restricted to a half-space rather than the whole space. For simplicity, it will be assumed that the structure can be accurately modeled by using the first three modes. Figure 1 plots the values of the magnitude of $M(\omega)$, denoted by the " + " marks, that were calculated from (2). The phase of the elements of $M(\omega)$ was either $0^{\circ}$ or $180^{\circ}$ and is not shown. In order to factor $M(\omega)$ to find the radiation filters, we first approximate it by a matrix of rational functions in the variable $s=j \omega$. The phase of the terms is easily matched by forcing the rational functions to have poles and zeros that are symmetric with respect to the imaginary axis in the $s$ plane. The magnitude response can be matched by a little trial and error fitting, and the result is shown by the continuous lines plotted in Fig. 1.

The $M(2,1)$ and $M(2,3)$ terms are identically equal to

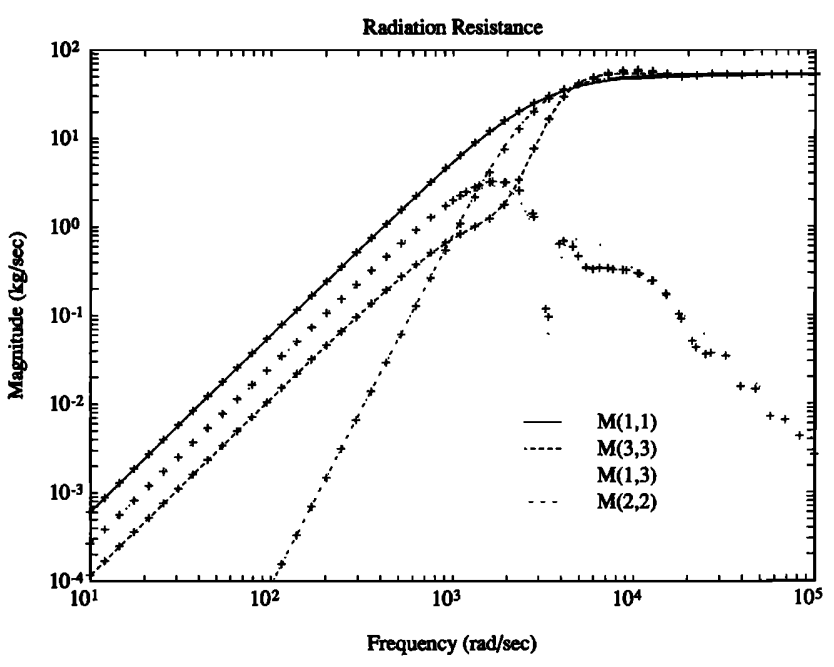

FIG. 1. Calculated values and rational approximation for $M(\omega)$.

zero and are not plotted. Thus, there is no interaction between the radiation of the second mode and the other two modes, but there is interaction between modes one and three at low frequencies. Physically, this is due to the fact that modes one and three, which are volumetric modes, look like monopoles at low frequencies and mode two, which is not a volumetric mode, looks like a dipole. Thus, over the entire half-plane being considered, there will be no net interaction in terms of radiated power between the dipole and the monopole, but the total power radiated by the two monopoles will depend on their relative magnitudes and phasing. At high frequencies, modes one and three cease to look like monopoles, and the interaction disappears.

\section{A. Nonradiating vibration}

Assume that the structural velocity components $V$ are given by the WSS harmonic process

$$
V(t)=V_{0} e^{-j\left(\omega_{0} t+\theta\right)},
$$

where $\theta$ is uniformly distributed between 0 and $2 \pi$. The power spectral density of this process is given by

$$
S_{V}(\omega)=2 \pi V_{0} V_{0}^{T^{*}} \delta\left(\omega+\omega_{0}\right) \text {. }
$$

The total power radiated can be computed from (1) to be

$$
\Pi=\operatorname{tr}\left(V_{0} V_{0}^{T^{*}} M\left(\omega_{0}\right)\right)=V_{0}^{T^{*}} M\left(\omega_{0}\right) V_{0} .
$$

Since this is a quadratic form, the minimum and maximum radiation due to a unit-norm excitation (i.e., $\left\|V_{0}\right\|=1$ ) can be computed from the eigenvalues of $M\left(\omega_{0}\right)$. For example, if $\omega_{0}=95.4 \mathrm{rad} / \mathrm{s}$,

$$
M(95.4)=\left[\begin{array}{ccc}
5.4906 & 0 & 2.4 \\
0 & 0.00736 & 0 \\
2.4 & 0 & 1.0491
\end{array}\right] \times 10^{-2}
$$

and has eigenvalues $6.5396 \times 10^{-2}, 7.3606 \times 10^{-5}$, $2.6998 \times 10^{-8}$ corresponding to the eigenvectors

$$
\left[\begin{array}{c}
0.916 \\
0 \\
0.401
\end{array}\right] ;\left[\begin{array}{l}
0 \\
1 \\
0
\end{array}\right] ;\left[\begin{array}{c}
0.401 \\
0 \\
-0.916
\end{array}\right] \text {. }
$$

The first eigenvalue corresponds to the maximum radiation 
possible with a unit-norm excitation. It consists of an inphase superposition of modes one and three with a relative weighting of 0.916 and 0.401 , respectively. As would be expected, this superposition maximizes the volumetric displacement of the vibration. The second eigenvalue represents the vibration of mode 2 alone, which produces significantly less radiation since it is not a volumetric mode. The third eigenvalue corresponds to the minimum radiation possible from a unit-norm excitation. It consists of an out-ofphase superposition of modes one and three with a relative weighting of 0.401 and -0.916 , respectively. This result depends on modeling the interaction of modes one and three through the off-diagonal terms in $M(\omega)$ and illustrates the fact that far-field radiation can be significantly suppressed without necessarily reducing the total vibration.

\section{B. Suppression of narrow-band excitation}

The linear shaping filter ( 3 ) that characterizes the disturbance is taken to be a second order filter with a center frequency of $95.4 \mathrm{rad} / \mathrm{s}$ and $1 \%$ damping. The transfer function is

$$
\frac{s}{s^{2}+0.95409 s+9103}
$$

and the frequency response is shown in Fig. 2. The frequency responses of the terms in the radiation filter are shown in Fig. 3. It is assumed that the disturbance enters at $0.36 \mathrm{~m}$ along the beam, which is near a node of the third mode. The actuator is located at $0.5 \mathrm{~m}$ along the beam, at a node of the second mode. Closed-loop controllers were designed for both vibration (with $Q=I$ ) and acoustic suppression. In this example, it was not desired to significantly penalize control effort and $\rho$ was set equal to $10^{-6}$. The signed magnitudes of the open and closed-loop velocities of the three modes, as well as the total radiated power, are given in Table I for a unit excitation at $95.4 \mathrm{rad} / \mathrm{s}$.

Given the position of the actuator, there is almost nothing that the vibration controller can do to reduce the overall vibration. The actuator does not affect the second mode and

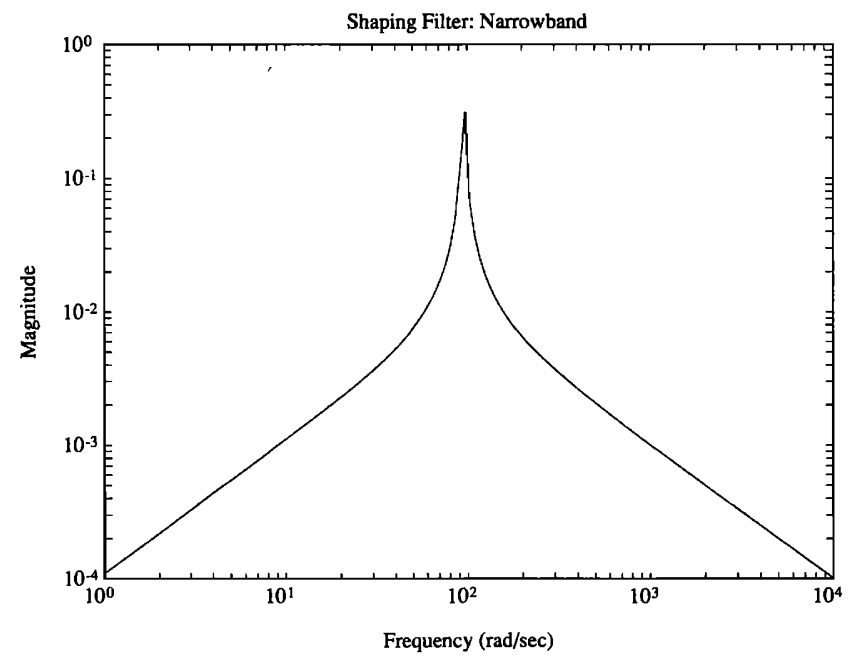

FIG. 2. Frequency response of narrowband shaping filter.

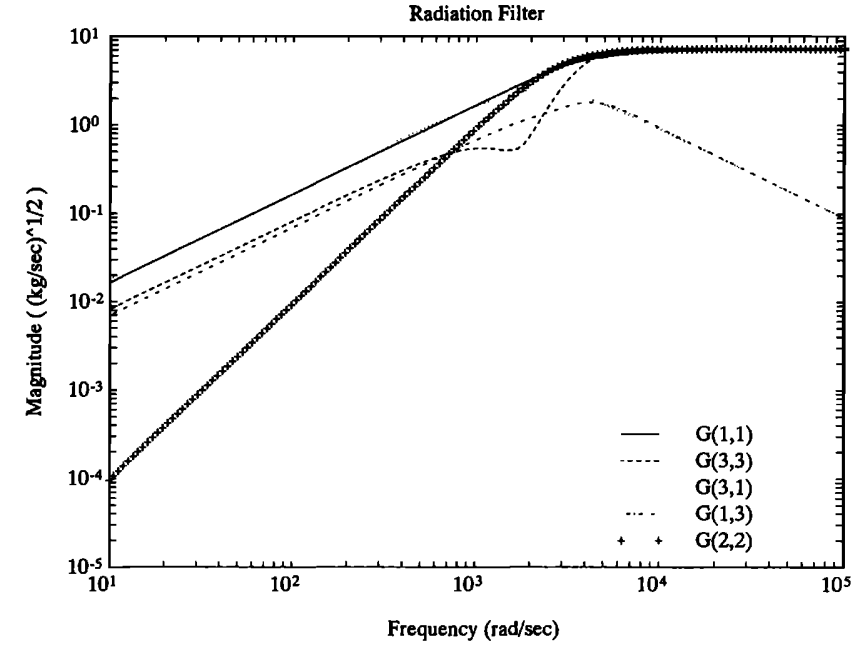

FIG. 3. Frequency response of radiation filter.

affects the first and third modes in opposite ways. Thus, although it suppresses the vibration of the first mode slightly, it is limited by the fact that the vibration of the third mode begins to increase. The acoustic controller, however, utilizes the acoustic interaction of modes one and three to suppress the far-field radiation. It actually increases the vibration of the first mode and greatly enhances the vibration of the third mode to create an overall vibration with about the same velocity ratio as the nonradiating vibration discussed previously. The result is that the radiated power is $24 \mathrm{~dB}$ below that of the uncontrolled and vibration-controlled cases.

The results for two other possible actuator placements are given in Tables II and III. For the case shown in Table II, the acoustic controller again suppresses the radiation by enhancing the vibration on the structure in such a way that the radiation from modes one and three cancel. The vibration controller reduces the vibration of modes one and two slightly, but at the expense of increasing the vibration of mode three. For the case shown in Table III, the acoustic controller suppresses the radiation by suppressing the vibration in modes one and three at the expense of the vibration in mode two which is greatly enhanced. The vibration controller reduces the vibration in mode two slightly while increasing the vibration in mode one slightly. This action actually increases the acoustic radiation.

\section{Suppression of broadband excitation}

The broadband disturbance is modeled by passing Gaussian white noise of unit intensity through a second-or-

TABLE I. Total radiated power and signed magnitudes of open and closedloop modal velocities for a unit disturbance at $95.4 \mathrm{rad} / \mathrm{s}$. Disturbance at $0.36 \mathrm{~m}$ and actuator at $0.50 \mathrm{~m}$.

\begin{tabular}{lcccc}
\hline \hline & Mode 1 & Mode 2 & Mode 3 & $\Pi$ \\
\hline Open-loop & 0.0144 & 0.0178 & -0.0020 & $1.01 e-5$ \\
Closed-loop vibration & 0.0138 & 0.0178 & +0.0020 & $1.19 e-5$ \\
Closed-loop acoustic & 0.0213 & 0.0178 & -0.0476 & $3.81 e-8$ \\
\hline \hline
\end{tabular}


TABLE II. Total radiated power and signed magnitudes of open and closed-loop modal velocities for a unit disturbance at $95.4 \mathrm{rad} / \mathrm{s}$. Disturbance at $0.36 \mathrm{~m}$ and actuator at $0.40 \mathrm{~m}$.

\begin{tabular}{lcccc}
\hline \hline & Mode 1 & Mode 2 & Mode 3 & $\Pi$ \\
\hline Open-loop & 0.0144 & 0.0178 & -0.002 & $1.01 e-5$ \\
Closed-loop vibration & 0.0115 & 0.0152 & +0.008 & $1.20 e-5$ \\
Closed-loop acoustic & 0.0349 & 0.0360 & -0.068 & $1.57 e-6$ \\
\hline \hline
\end{tabular}

der Chebyshev bandpass shaping filter. The frequency responses of several of these filters with different passbands are shown in Fig. 4. The disturbance is again taken to enter at $0.36 \mathrm{~m}$ along the beam and the actuator is located at $0.5 \mathrm{~m}$ along the beam. To more accurately model the real situation, we also assume that each mode of the beam has $1 \%$ damping.

The frequency responses of the three vibration modes from the disturbance input to the modal velocity, for both uncontrolled and controlled cases, are shown in Fig. 5(a)(c) for a disturbance bandwidth equal to $50 \mathrm{rad} / \mathrm{s}$ centered at $70.7 \mathrm{rad} / \mathrm{s}$. The optimal (LQG) acoustic controller employs the same mechanism used for sinusoidal suppression. It tends to suppress the vibration of the first mode and enhance the vibration of the third mode such that in the entire disturbance passband a desired velocity ratio of mode 1 and mode 3 is obtained, and the radiation of mode 1 and mode 3 cancel.

At the frequency $\omega_{0}=95.4 \mathrm{rad} / \mathrm{s}$, the eigenvector $\left[\begin{array}{lll}0.916 & 0 & 0.401\end{array}\right]^{T}$ of $M\left(\omega_{0}\right)$ represents the direction of maximum radiation, which consists of an in-phase superposition of modes one and three. If the eigenvector does not change significantly over the entire disturbance passband, which is true for our example above, the closed-loop frequency response from the disturbance input to a fictitious output consisting of a weighted sum of the modal velocities ( 0.916 for mode 1,0 for mode 2 and 0.401 for mode 3 ) can be used to model the frequency response from the disturbance to the maximally radiating velocity pattern. This frequency response is shown in Fig. 5(d) and can be seen to exhibit a notch in the disturbance passband, as would be expected.

If we let $H(j \omega)$ represent the transfer function of the cascade of the shaping filter, closed-loop model and radiation filter, then the expected value of the radiated power is given by

$$
\frac{1}{2 \pi} \int_{-\infty}^{+\infty}|H(j \omega)|^{2} d \omega .
$$

The results of this computation are shown in Table IV. The

TABLE III. Total radiated power and signed magnitudes of open and closed-loop modal velocities for a unit disturbance at $95.4 \mathrm{rad} / \mathrm{s}$. Disturbance at $0.36 \mathrm{~m}$ and actuator at $0.64 \mathrm{~m}$.

\begin{tabular}{lcccc}
\hline \hline & Mode 1 & Mode 2 & Mode 3 & $\Pi$ \\
\hline Open-loop & 0.0144 & 0.0178 & -0.002 & $1.01 e-5$ \\
Closed-loop vibration & 0.0172 & 0.0143 & -0.002 & $1.44 e-5$ \\
Closed-loop acoustic & 0.0014 & 0.0338 & -0.000 & $1.79 e-7$ \\
\hline \hline
\end{tabular}

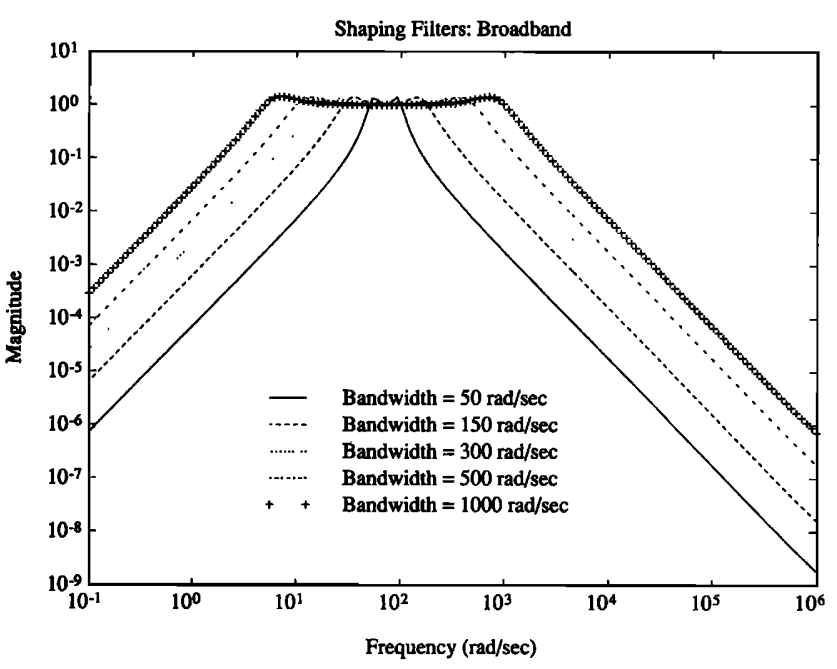

FIG. 4. Frequency responses to broadband shaping filters.

acoustic controller provides $10 \mathrm{~dB}$ more suppression than the vibration controller. If the control effort is penalized less, the acoustically controlled system can suppress the radiation by at most $17 \mathrm{~dB}$, as shown in Table $\mathrm{V}$, while the vibrationcontrolled system does not give increased suppression of radiated power.

Plots of the time responses of the three systems to sample inputs are given in Fig. 6(a)-(c). It can be seen that the acoustic controller excites mode three more than the vibration controller, as would be expected from the narrowband results. Since mode three is out of phase with mode one, this reduces the acoustic radiation by reducing the volumetric displacement of the overall vibration.

The comparison between acoustic and vibration controllers for disturbances with different passbands is shown in Table V. Control effort is penalized by $\rho=1 e-12$. As the bandwidth of the disturbance gets broader, the acoustic controller provides less of an advantage over the vibration controller. As can be seen from Fig. 1, for frequencies higher than $1000 \mathrm{rad} / \mathrm{s}$ the modes begin to radiate independently and the goals of vibration suppression and acoustic suppression become identical.

The control design method that has been discussed is not limited to a single actuator. The performance resulting from an added actuator placed at $0.25 \mathrm{~m}$ along the beam is shown in Fig. 7 for the 50-rad/s disturbance bandwidth example considered previously $(\rho=1 e-12)$. Compared to the single actuator result, the notch in the disturbance passband is not only deeper, but the spike due to the third mode is eliminated since it is now possible to control modes 1 and 3 independently.

\section{Simplifying the radiation filters}

The order of the system used to fit the $M(\omega)$ data was 24. This resulted in a 12th-order radiation filter, which means that the order of the acoustic controller was 12 higher than that of the vibration controller. For the low-frequency case, it seems reasonable to model only the low frequency 

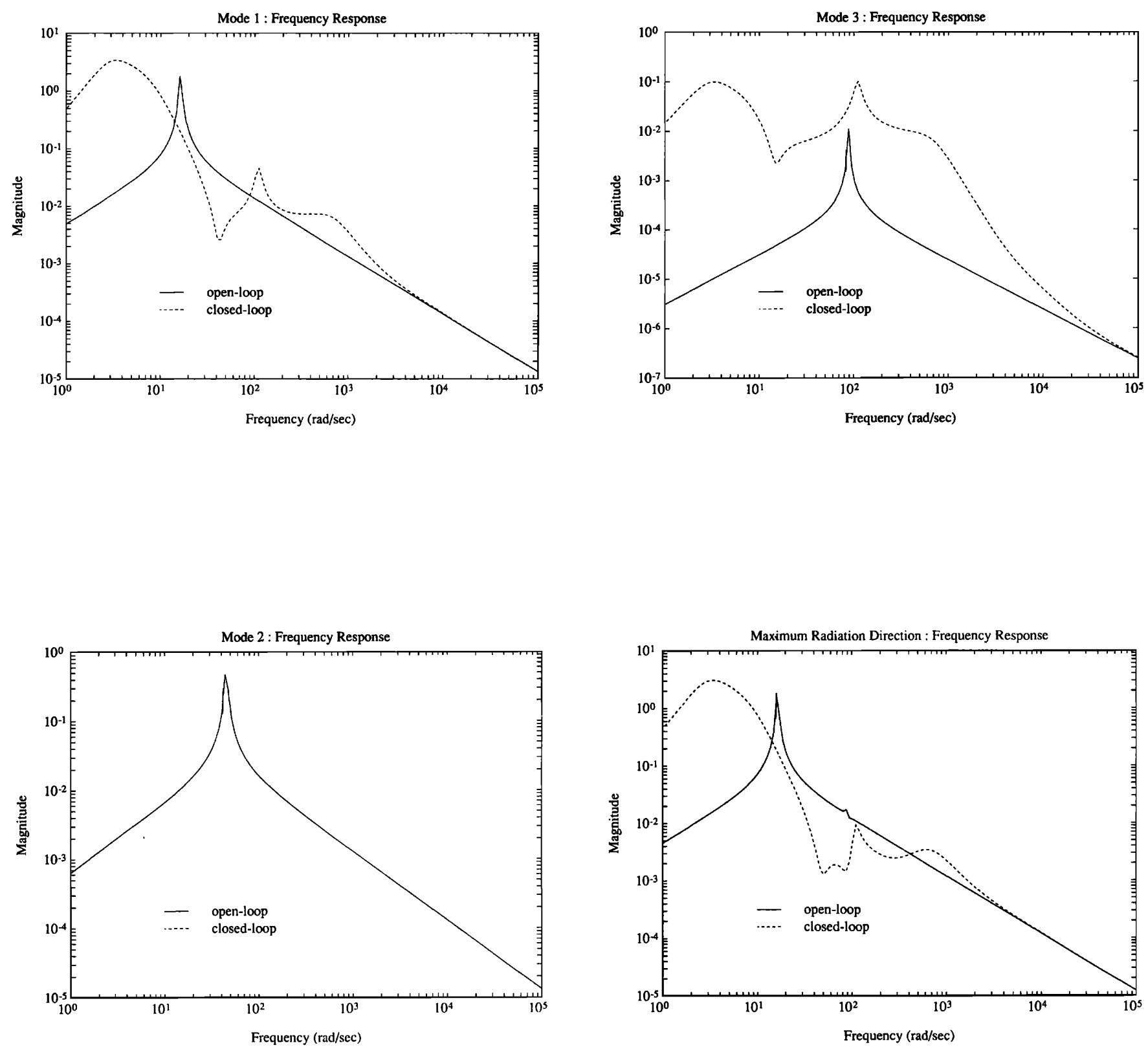

FIG. 5. (a) Frequency response from disturbance input to mode 1 velocity. (b) Frequency response from disturbance input to mode 2 velocity. (c) Frequency response from disturbance input to mode 3 velocity. (d) Frequency response from disturbance input to maximally radiating velocity distribution.

portion of $M(\omega)$. By dropping out the $(2,2)$ component completely, and modeling the other terms of $M(\omega)$ by second-order (to get the correct slope) high-pass filters, the order of the approximation can be reduced to eight, resulting

TABLE IV. Total radiated power due to disturbance with $50-\mathrm{rad} / \mathrm{s}$ bandwidth and $\rho=1 e-6$.

\begin{tabular}{ll}
\hline \hline System & Radiated power \\
\hline Open loop & $3.3639 e-4$ \\
Closed-loop vibration & $1.9137 e-4$ \\
Closed-loop acoustic & $1.8449 e-5$ \\
Closed-loop acoustic (reduced) & $1.8450 e-5$ \\
\hline
\end{tabular}

TABLE V. Total radiated power due to broadband disturbances with different bandwidths and $\rho=1 e-12$.

\begin{tabular}{rlll}
\hline \hline $\begin{array}{l}\text { Bandwidth } \\
(\mathrm{rad} / \mathrm{s})\end{array}$ & Open-loop & $\begin{array}{c}\text { Closed-loop } \\
\text { vibration }\end{array}$ & $\begin{array}{c}\text { Closed-loop } \\
\text { acoustic }\end{array}$ \\
\hline 50 & $3.3639 e-4$ & $1.9151 e-4$ & $4.6816 e-6$ \\
150 & $1.2 e-3$ & $7.0749 e-4$ & $2.6021 e-5$ \\
300 & $7.2 e-3$ & $1.2 e-3$ & $4.2026 e-5$ \\
500 & $1.08 e-2$ & $1.7 e-3$ & $1.1198 e-4$ \\
1000 & $1.11 e-2$ & $3.0 e-3$ & $6.1113 e-4$ \\
4000 & $2.54 e-2$ & $1.13 e-2$ & $9.6 e-3$ \\
8000 & $3.12 e-2$ & $1.63 e-2$ & $1.52 e-2$ \\
\hline \hline
\end{tabular}



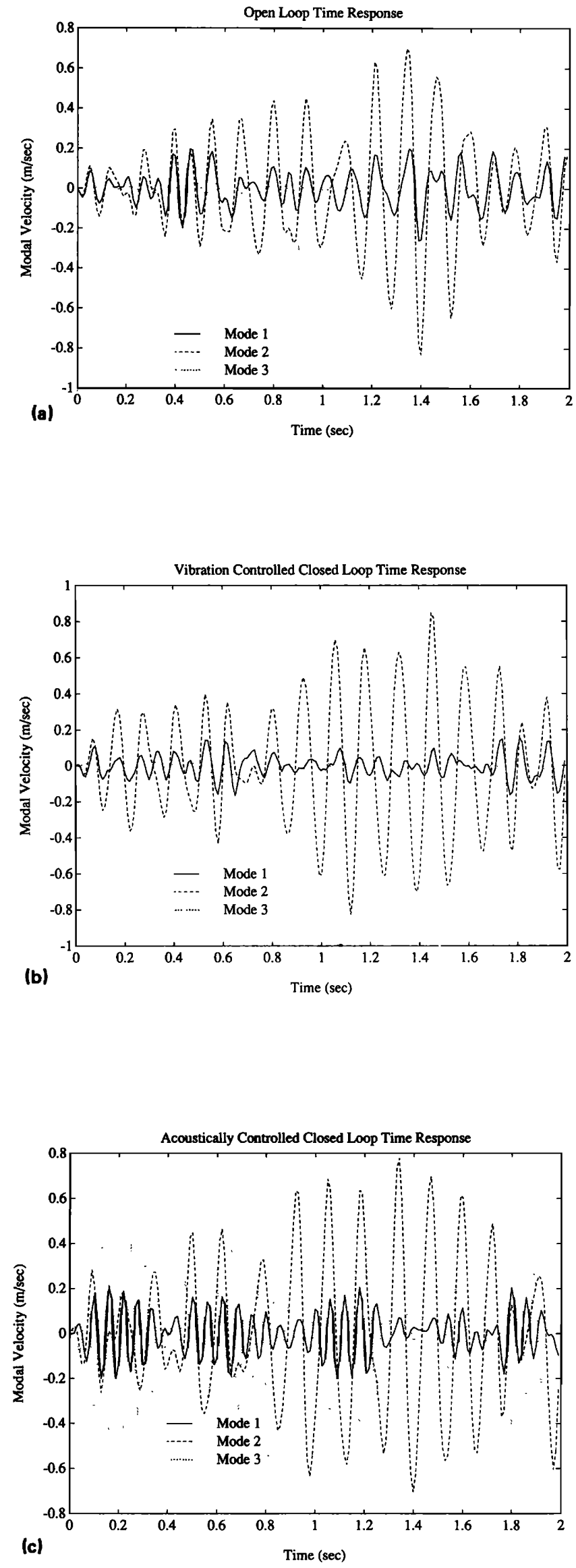

FIG. 6. (a) Open-loop time response to sample input. (b) Time response to sample input with vibration controller. (c) Time response to sample input with acoustic controller.

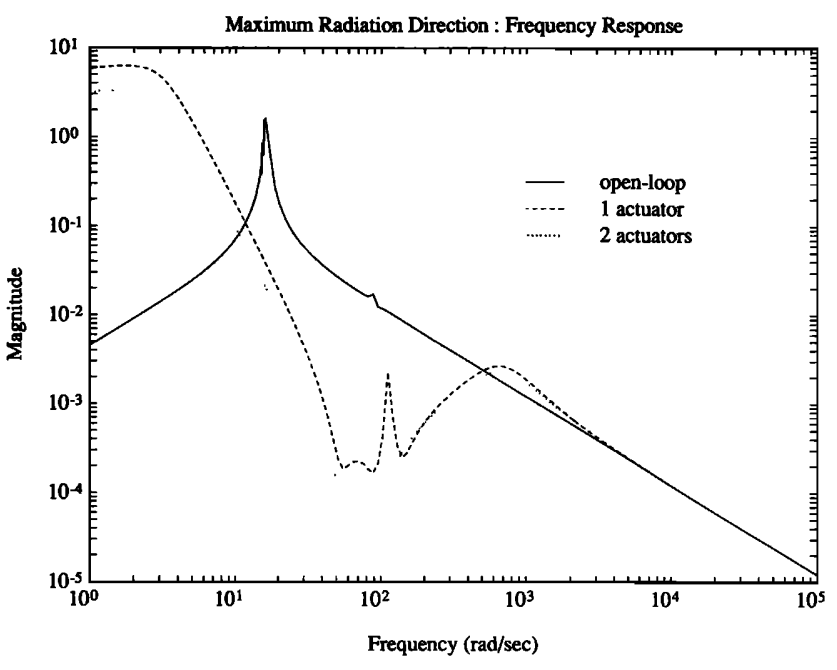

FIG. 7. Frequency responses from disturbance input to maximally radiating velocity distribution for 1 and 2 actuators $(\rho=1 E-12)$.

in a fourth-order radiation filter. The second-order fits and the frequency response of the resulting radiation filter are shown in Figs. 8 and 9. Redoing the calculation of the previous section to compute the expected value of the radiated power when the reduced-order acoustic controller is used results in the last entry of Table IV. Clearly there is no practical difference between the full-order and reduced-order controllers for low-frequency ( 10 to $500 \mathrm{rad} / \mathrm{s}$ ) disturbances.

\section{CONCLUSIONS}

In this paper we have developed a method for estimating the total radiated power. By using this estimate as a cost function for an optimal control problem, the acoustic suppression problem for persistent disturbances can be cast as a standard linear-quadratic-Gaussian problem and an optimal feedback controller can be computed to minimize the radiated power. This methodology was applied to a simple example and it was shown that the acoustic controller significant-

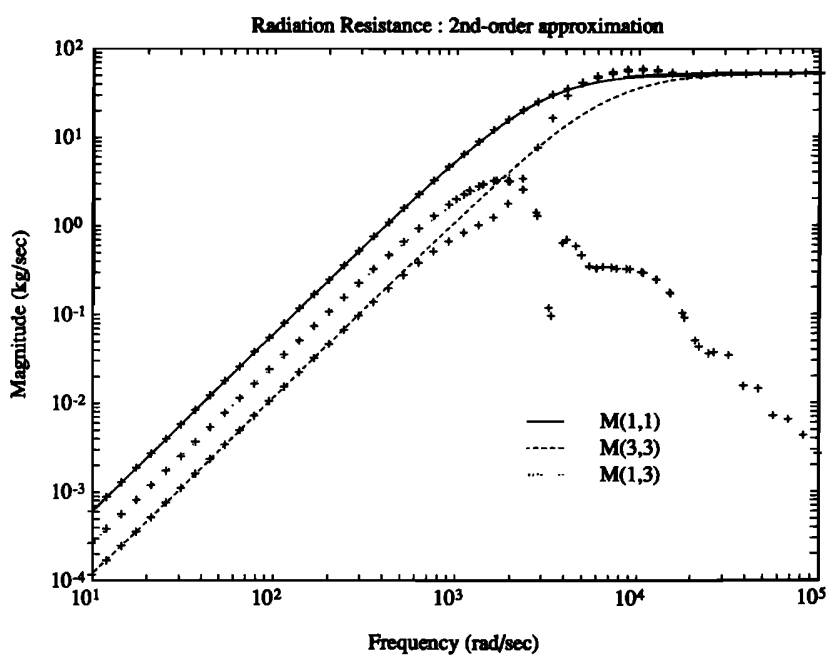

FIG. 8. Low frequency approximations to $M(\omega)$. 


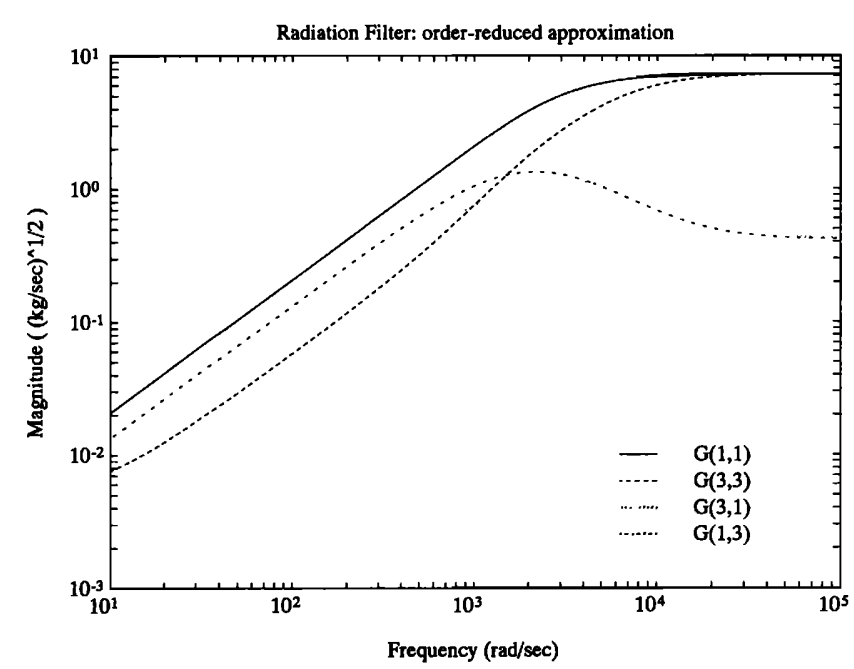

FIG. 9. Frequency response of reduced-order radiation filter.

ly outperformed a vibration controller for harmonic disturbances. At times, the acoustic controller actually increased the vibration while reducing the radiation, taking advantage of vibration patterns with very low radiation efficiencies. For broadband disturbances in the low-frequency region, the acoustic controller again outperformed a vibration controller. For broadband disturbances with the majority of their energy concentrated in the high-frequency region, where the modes radiate independently, there is very little difference between the acoustic and vibration controllers.

\section{ACKNOWLEDGMENT}

This work was supported under DARPA/ONR Contract N00014-88-K-0721.

'D. R. Morgan, "An adaptive modal-based active control system," J. Acoust. Soc. Am. 89, 128-256 (1991).

${ }^{2}$ C. R. Fuller, "Active control of sound transmission/radiation from elastic plates by vibration inputs: I. Analysis," J. Sound Vib. 136, 1-15 (1990).

${ }^{3}$ L. J. Eriksson, "Development of the filtered- $U$ algorithm for active noise control," J. Acoust. Soc. Am. 89, 257-265 (1991).

${ }^{4}$ L. Meirovitch and S. Thangjitham, "Active Control of Sound Radiation Pressure," J. Vib. Acoust. Stress Reliability Design 112, 237-244 (1990).

${ }^{5} \mathrm{~L}$. Meirovitch and S. Thangjitham, "Control of sound radiation from submerged plates," J. Acoust. Soc. Am. 88, 402-407 (1990).

${ }^{6}$ W. T. Baumann, W. R. Saunders, and H. H. Robertshaw, "Active Suppression of Acoustic Radiation from Impulsively Excited Structures," J. Acoust. Soc. Am. 90, 3202-3208 (1991).

${ }^{7}$ C. E. Wallace, "The Radiation Resistance of a Baffled Beam," J. Acoust. Soc. Am. 51, 936-945 (1972).

${ }^{8}$ J. M. Maciejowski, Multivariable Feedback Design (Addison-Wesley, Reading, MA, 1989). 